Introduction Early detection of oesophageal adenocarcinoma (AC) or high grade dysplasia (HGD) improves prognosis but may missed at endoscopy.

Hypothesis Glycosylation alterations in the progression to $\mathrm{AC}$ could be detected using lectins to allow endoscopic imaging with a wide field of view.

Aims (1) Determine if and when glycan gene expression and lectin binding alters in progression to AC. (2) Determine whether fluorescently labelled lectins can detect dysplastia in biopsies and at endoscopy.

Methods Gene set enrichment analysis of glycan pathways was applied to gene expression data from squamous oesophagus (NE), Barrett's oesophagus (BE), LGD and HGD ( $n=57)$. Human samples $(\mathrm{n}=21)$ were applied to evanescent and ratiometric lectin arrays ( $n=45$ and 78). External validation used lectin histochemistry $(n=80)$. Fresh whole biopsy lectin staining was performed at the bedside $(n=33)$ using WGA-680 and assessed using IVIS 200. Whole oesophagus imaging used WGA-488 with endoscopic excitation at $395-475 \mathrm{~nm}$ and detection at 500-630 nm. Specificity of WGA binding was confirmed using $\mathrm{N}$-acetyl glucosamine in a competition assay and by preincubation with $\alpha(2 \rightarrow 3,6,8,9)$ neuraminidase. Statistics: Jonckheere-Terpstra test for trend, Bonferroni correction to ANOVA and a one-sided paired t test for the specificity assays.

Results Coordinated changes in glycan expression occur at every stage of the progression to AC ( $p<0.05)$. Ratiometric lectin array data identified a cluster of lectins with high binding to NE which decreased with progression to AC ( $p<0.000001)$ and evanescent array data confirmed 4 lectins from this group with the most significant binding differences in progression $(p<0.05)$ : WGA, HPA, AOL and TJA-I. WGA, HPA and AOL all successfully externally validated using histochemistry $(p<0.01)$. Whole-biopsy WGA binding was diminished $(p<0.0001)$ in biopsies containing dysplasia. Differences in WGA binding were identified in the intact oesophagus using standard endoscopic equipment and fluorescent areas correlated with the areas of high grade dysplasia (not visible with white light endoscopy) mapped in the surgical resection specimen.

Conclusion Surface glycans alter in the progression from $\mathrm{BE}$ to $\mathrm{AC}$ and lead to altered lectin binding. WGA validated to the point of successful endoscopic visualisation of high grade dysplasia. Further studies are needed to determine the sensitivity and specificity of this molecular imaging tool in vivo.

Competing interests None.

Keywords Adenocarcinoma, Barrett's oesophagus, Endoscopic, Glycans, Lectin, Molecular imaging, Wheat germ agglutinin.

\section{PWE-098 MOLECULAR IMAGING VIA NOVEL APPLICATION OF FLUORESCENT LECTINS PERMITS RAPID ENDOSCOPIC IDENTIFICATION OF DYSPLASIA IN BARRETT'S OESOPHAGUS}

doi:10.1136/gut.2011.239301.361
E L Bird-Lieberman, ${ }^{1, *}$ A A Neves, ${ }^{2}$ P Lao-Sirieix, ${ }^{1}$ M O'Donovan, ${ }^{3}$ L B Lovat, ${ }^{4}$ L K Mahal, ${ }^{5} \mathrm{~K}$ M Brindle, ${ }^{2} \mathrm{R}$ C Fitzgerald ${ }^{1}{ }^{1}$ MRC Cancer Cell Unit, Hutchison-MRC Research Centre, Cambridge, UK; ${ }^{2}$ Cancer Research UK Cambridge Research Institute and University Department of Biochemistry, University of Cambridge, Cambridge, UK; ${ }^{3}$ Department of Pathology, Cambridge University Hospital, Cambridge, UK; ${ }^{4}$ Department of Gastroenterology, University College London, London, UK; ${ }^{5}$ Department of Chemistry, New York University, New York, New York, USA 